\title{
C-projection and monopole condensation in QCD
}

\author{
Y. M. Cho \\ Administration Building 310-4 \\ Konkuk University, Seoul 143-701, Korea \\ and \\ School of Physics and Astronomy \\ Seoul National University, Seoul 151-747, Korea \\ ymcho7@konkuk.ac.kr \\ Published 16 April 2014
}

\begin{abstract}
We show that the monopole condensation is responsible for the confinement. To demonstrate this we present a new gauge invariant integral expression of the one-loop QCD effective action which has no infra-red divergence, and show that the color reflection invariance ("the C-projection") assures the gauge invariance and the stability of the monopole condensation.
\end{abstract}

Keywords: Abelian dominance; dual Meissner effect; color reflection invariance; C-parity; C-projection; monopole condensation; color confinement.

PACS Numbers: 12.38.-t, 12.38.Aw, 11.15.-q, 11.15.Tk

\section{Introduction}

One of the most challenging problems in theoretical physics is the confinement problem in quantum chromodynamics (QCD). Two outstanding conjectures of the confinement mechanism are the monopole condensation and the Abelian dominance. ${ }^{1-5}$ It has long been argued that the confinement in QCD can be triggered by the monopole condensation. ${ }^{1-3}$ Indeed, if one assumes the monopole condensation, one can easily argue that the ensuing dual Meissner effect could guarantee the color confinement. To prove the monopole condensation, however, has been extremely difficult.

A natural way to establish the monopole condensation in QCD is to show that the quantum fluctuation triggers a phase transition similar to the dimensional transmutation observed in massless scalar QED. ${ }^{6}$ There have been many attempts to

This is an Open Access article published by World Scientific Publishing Company. It is distributed under the terms of the Creative Commons Attribution 3.0 (CC-BY) License. Further distribution of this work is permitted, provided the original work is properly cited. 
demonstrate this. Savvidy has first calculated the effective action of SU(2) QCD in the presence of an ad hoc color magnetic background, and has almost "proved" the magnetic condensation. ${ }^{7}$

Unfortunately, the subsequent calculation repeated by Nielsen and Olesen showed that the effective action has an extra imaginary part which destablizes the magnetic condensation. This is known as the instability of the "Savvidy-NielsenOlesen (SNO) vacuum" ${ }^{8-10}$ The origin of this instability can be traced to the tachyonic modes in the functional determinant of the gluon loop integral.

But in physics we encounter the tachyons when we do something wrong. For example, in spontaneous symmetry breaking we have tachyons when we choose the false vacuum. Similarly, in Neveu-Schwarz-Ramond (NSR) string we have the tachyonic vacuum when we do not make the theory supersymmetric and modular invariant with the Gliozzi-Scherk-Olive (GSO) projection. ${ }^{11,12}$ The question here is how to remove the tachyonic modes in the gluon functional determinant, and how to justify that.

We emphasize, however, that the most seriuos defect of the SNO vacuum is not that it is unstable but that it is not gauge invariant. So even if the SNO vacuum were made stable, it can not be the QCD vacuum. This is why the so-called "Copenhagen vacuum" is proposed. 8

On the other hand the Abelian dominance proposed by 'tHooft asserts that the "Abelian part" of QCD must be responsible for the confinement. ${ }^{4,5}$ The justification of this is that the colored (non-Abelian) gluons can not play any role in the confinement, because they (just like the quarks) themselves have to be confined. So, only the neutral (Abelian) gluons, if at all, could possibly contribute to the confinement. In this sense, this conjecture must be true. In fact we can show explicitly that the Abelian part of the potential is responsible for the confining force in QCD. ${ }^{13}$

The Abelian dominance has become very popular and widely been studied in lattice QCD. ${ }^{5}$ However, it has serious drawbacks. The popular way to obtain the Abelian part with "the maximal Abelian gauge" is gauge dependent. More seriously, this does not tell exactly what constitutes the Abelian part. Obviously the Abelian part must contain the trivial Maxwell-type Abelian potential, but this U(1) potential is not supposed to produce the confinement. So the Abelian part must contain something else, but the maximal Abelian gauge does not specify what is that. So, even if we prove the Abelian dominance, we can not tell what is really responsible for the confinement.

The gauge independent Abelian projection defined by the magnetic isometry tells exactly what is the Abelian part. ${ }^{2,3}$ It tells that the Abelian potential is made of two parts, the non-topological Maxwell part and the topological monopole part. So, unlike the Abelian projection based on the maximal Abelian gauge, this allows us to test not only the Abelian dominance but also the monopole condensation gauge independently and thus pinpoint exactly what is responsible for the confinement.

Implementing the gauge independent Abelian projection on the lattice, the SNU-Konkuk and the KEK-Chiba Lattice Collaborations independently have 


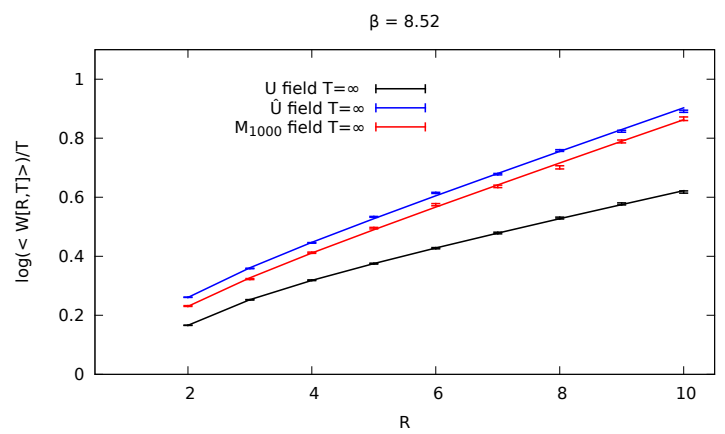

Fig. 1. The lattice QCD result which establishes the monopole dominance in the linear confining force gauge independently. Here the confining forces marked by red, blue, and black lines are obtained with the full potential, the Abelian potential, and the monopole potential, respectively.

demonstrated that the confining force comes from the monopole part of the Abelian projection. ${ }^{14,15}$ The SNU-Konkuk result of SU(3) QCD is shown in Fig. 1. The lattice result, however, does not prove the monopole condensation. It only tells that the monopole is responsible for the confinement.

The purpose of this paper is to calculate the one-loop QCD effective action in the presence of the monopole background, and establish the stable monopole condensation in $\mathrm{SU}(2) \mathrm{QCD}$ theoretically. First, we show that the SNO vacuum violates not only the gauge invariance but also the parity, and prove that only the monopole background conserve both gauge symmetry and parity. Second, imposing the color reflection invariance (the C-parity) on the gluon functional determinant which assures the gauge invariance, we obtain a new infra-red finite integral expression of QCD effective action. From this we show that a stable monopole condensation takes place and becomes the true vacuum of QCD. In particular we show that, just as the GSO projection (the G-parity) removes the tachyonic vacuum in string theory, the C-parity in QCD removes the tachyons and assures the stability of the monopole condensation. This establishes the dimensional transmutation by the monopole condensation in QCD.

\section{RCD, ECD, and ACD}

To prove the monopole condensation gauge independently we have to understand the Abelian decomposition of QCD first. For simplicity consider the SU(2) QCD and let $\left(\hat{n}_{1}, \hat{n}_{2}, \hat{n}_{3}\right)$ be a righthanded orthonormal basis. Choose $\hat{n}=\hat{n}_{3}$ to be the Abelian direction and impose the magnetic isometry to the gauge potential $\vec{A}_{\mu}$ to make the Abelian projection

$$
\begin{gathered}
D_{\mu} \hat{n}=\partial_{\mu} \hat{n}+g \vec{A}_{\mu} \times \hat{n}=0, \\
\vec{A}_{\mu} \rightarrow \hat{A}_{\mu}=A_{\mu} \hat{n}-\frac{1}{g} \hat{n} \times \partial_{\mu} \hat{n}=\mathcal{A}_{\mu}+\mathcal{C}_{\mu},
\end{gathered}
$$




$$
\mathcal{A}_{\mu}=A_{\mu} \hat{n}, \quad \mathcal{C}_{\mu}=-\frac{1}{g} \hat{n} \times \partial_{\mu} \hat{n}, \quad A_{\mu}=\hat{n} \cdot \vec{A}_{\mu}
$$

Notice that $\mathcal{C}_{\mu}$, when $\hat{n}=\hat{r}$, describes the Wu-Yang monopole. ${ }^{16,17}$ This tells that the potential $\hat{A}_{\mu}$ which leaves $\hat{n}$ invariant under the parallel transport is made of the "naive" Abelian part $\mathcal{A}_{\mu}$ and the monopole part $\mathcal{C}_{\mu}$.

To understand the meaning of this dual structure of $\hat{A}_{\mu}$, notice that

$$
\begin{gathered}
\hat{F}_{\mu \nu}=\partial_{\mu} \hat{A}_{\nu}-\partial_{\nu} \hat{A}_{\mu}+g \hat{A}_{\mu} \times \hat{A}_{\nu}=\left(F_{\mu \nu}+H_{\mu \nu}\right) \hat{n} \\
F_{\mu \nu}=\partial_{\mu} A_{\nu}-\partial_{\nu} A_{\mu} \\
H_{\mu \nu}=-\frac{1}{g} \hat{n} \cdot\left(\partial_{\mu} \hat{n} \times \partial_{\nu} \hat{n}\right)=\partial_{\mu} \tilde{C}_{\nu}-\partial_{\nu} \tilde{C}_{\mu} \\
\tilde{C}_{\mu}=-\frac{1}{g} \hat{n}_{1} \cdot \partial_{\mu} \hat{n}_{2}
\end{gathered}
$$

where $\tilde{C}_{\mu}$ becomes exactly the Dirac's monopole potential. ${ }^{2,3}$ This tells that $\mathcal{A}_{\mu}$ and $\mathcal{C}_{\mu}$ (or equivalently $A_{\mu}$ and $\tilde{C}_{\mu}$ ) represent the non-topological Maxwellian "electric" potential and the topological Diracian "magnetic" potential.

With the Abelian projection we have the Abelian decomposition known as the Cho-Duan-Ge (CDG) decomposition or the Cho-Faddeev-Niemi (CFN) decomposition, ${ }^{18-20}$

$$
\vec{A}_{\mu}=\hat{A}_{\mu}+\vec{X}_{\mu}, \quad \vec{X}_{\mu}=X_{\mu}^{1} \hat{n}_{1}+X_{\mu}^{2} \hat{n}_{2}
$$

Under the infinitesimal gauge transformation we have

$$
\begin{gathered}
\delta A_{\mu}=\frac{1}{g} \hat{n} \cdot \partial_{\mu} \vec{\alpha}, \quad \delta \hat{A}_{\mu}=\frac{1}{g} \vec{D}_{\mu} \vec{\alpha}, \\
\delta \vec{X}_{\mu}=-\vec{\alpha} \times \vec{X}_{\mu} .
\end{gathered}
$$

So $\hat{A}_{\mu}$ by itself describes an $\mathrm{SU}(2)$ connection which enjoys the full $\mathrm{SU}(2)$ gauge degrees of freedom. Moreover, $\vec{X}_{\mu}$ becomes gauge covariant. Most importantly, the decomposition is gauge independent. Once $\hat{n}$ is given, the decomposition uniquely defines $\hat{A}_{\mu}$ and $\vec{X}_{\mu}$, independent of the choice of gauge. ${ }^{2,3}$

With the Abelian decomposition we obtain the restricted QCD (RCD) which describes the Abelian sub-dynamics of $\mathrm{QCD}^{2,3}$

$$
\begin{gathered}
\mathcal{L}_{R C D}=-\frac{1}{4} \hat{F}_{\mu \nu}^{2}=-\frac{1}{4} F_{\mu \nu}^{2} \\
+\frac{1}{2 g} F_{\mu \nu} \hat{n} \cdot\left(\partial_{\mu} \hat{n} \times \partial_{\nu} \hat{n}\right)-\frac{1}{4 g^{2}}\left(\partial_{\mu} \hat{n} \times \partial_{\nu} \hat{n}\right)^{2} .
\end{gathered}
$$

It has the full $\mathrm{SU}(2)$ gauge freedom, in spite of the fact that it is simpler than QCD. Because of this it retains the non-Abelian topology of QCD, and contains the monopole degrees explicitly. This makes RCD an ideal flatform for us to discuss the monopole dynamics gauge independently. 
Furthermore, with (3) we have the extended QCD (ECD),

$$
\begin{gathered}
\mathcal{L}_{E C D}=-\frac{1}{4} \vec{F}_{\mu \nu}^{2}=-\frac{1}{4} \hat{F}_{\mu \nu}^{2}-\frac{1}{4}\left(\hat{D}_{\mu} \vec{X}_{\nu}-\hat{D}_{\nu} \vec{X}_{\mu}\right)^{2} \\
-\frac{g}{2} \hat{F}_{\mu \nu} \cdot\left(\vec{X}_{\mu} \times \vec{X}_{\nu}\right)-\frac{g^{2}}{4}\left(\vec{X}_{\mu} \times \vec{X}_{\nu}\right)^{2} .
\end{gathered}
$$

This shows that QCD can be viewed as RCD made of the binding gluons, which has the colored valence gluons as its source. ${ }^{2,3}$ Notice, however, that ECD has more gauge symmetry. In addition to the classical (slow) gauge symmetry of QCD, it has the extra quantum (fast) gauge symmetry. This is because the decomposition (3) automatically put (6) to the background field formalism which doubles the gauge symmetry. ${ }^{21,22}$

With this we can actually Abelianize ECD and have the Abelianized QCD $(\mathrm{ACD}), 2,3$

$$
\begin{aligned}
& \mathcal{L}_{A C D}=-\frac{1}{4} G_{\mu \nu}^{2}-\frac{1}{2}\left|\hat{D}_{\mu} X_{\nu}-\hat{D}_{\nu} X_{\mu}\right|^{2} \\
&+i g G_{\mu \nu} X_{\mu}^{*} X_{\nu}-\frac{1}{2} g^{2}\left[\left(X_{\mu}^{*} X_{\mu}\right)^{2}-\left(X_{\mu}^{*}\right)^{2}\left(X_{\nu}\right)^{2}\right] \\
& G_{\mu \nu}=\partial_{\mu} B_{\nu}-\partial_{\nu} B_{\mu}=F_{\mu \nu}+H_{\mu \nu} \\
& \hat{D}_{\mu} X_{\nu}=\left(\partial_{\mu}+i g B_{\mu}\right) X_{\nu} \\
& B_{\mu}=A_{\mu}+\tilde{C}_{\mu}, \quad X_{\mu}=\frac{1}{\sqrt{2}}\left(X_{\mu}^{1}+i X_{\mu}^{2}\right)
\end{aligned}
$$

Formally this is what we can obtain from the Yang-Mills Lagrangian with $\vec{A}_{\mu}=$ $\left(X_{\mu}^{1}, X_{\mu}^{2}, B_{\mu}\right)$. But this is a gauge dependent Abelianization. In comparison (7) is gauge independent, because here we have never fixed the gauge to obtain ACD.

This ACD, moreover, is not Abelian because it retains the full non-Abelian gauge symmetry. Actually it has not only the classical gauge symmetry but also the quantum gauge symmetry. ${ }^{23,24}$

Now we show that RCD, ECD, and ACD have a new discrete symmetry called the color reflection invariance which makes them fundamentally different from QED. To see this notice that the Abelian decomposition is not uniquely determined even after we select the Abelian direction. Consider the the color reflection, the gauge transformation which inverts the color direction (the $\pi$-rotation along $\hat{n}_{1}$ ),

$$
\left(\hat{n}_{1}, \hat{n}_{2}, \hat{n}\right) \rightarrow\left(\hat{n}_{1},-\hat{n}_{2},-\hat{n}\right) .
$$

Obviously the physics should not change under this change of basis. On the other hand, the isometry condition (1) is insensitive to this change. So we have two different Abelian decompositions imposing the same isometry using two different bases, without changing the physics. This tells that the color reflection (8) which originally was introduced as a gauge transformation now becomes a discrete symmetry of $R C D, E C D$, and $A C D$ after the Abelian decomposition. ${ }^{2,3}$ 
To amplify this notice that, under the color reflection we have

$$
\begin{gathered}
\hat{A}_{\mu} \rightarrow \hat{A}_{\mu}^{(c)}=-A_{\mu} \hat{n}-\frac{1}{g} \hat{n} \times \partial_{\mu} \hat{n}=-\mathcal{A}_{\mu}+\mathcal{C}_{\mu}, \\
A_{\mu} \rightarrow A_{\mu}^{(c)}=-A_{\mu}, \quad \tilde{C}_{\mu} \rightarrow \tilde{C}_{\mu}^{(c)}=\tilde{C}_{\mu} \\
X_{\mu} \rightarrow X_{\mu}^{(c)}=X_{\mu}^{*}=\frac{X_{\mu}^{1}-i X_{\mu}^{2}}{\sqrt{2}} \\
\hat{F}_{\mu \nu} \rightarrow \hat{F}_{\mu \nu}^{(c)}=\left(-F_{\mu \nu}+H_{\mu \nu}\right) \hat{n} .
\end{gathered}
$$

Clearly the valence gluon change the color. Moreover, $\mathcal{A}_{\mu}$ and $\mathcal{C}_{\mu}$ (as well as $A_{\mu}$ and $\tilde{C}_{\mu}$ ) transform oppositely under (8). In particular the Diracian magnetic (topological) part $\mathcal{C}_{\mu}$ (and $H_{\mu \nu}$ ) remains invariant under the color reflection while the Maxwellian electric (non-topological) part $\mathcal{A}_{\mu}$ (and $F_{\mu \nu}$ ) changes the signature.

This assures that changing the color of the valence gluons does not change the physics, which is another way of saying that colored objects are unphysical. This, of course, amounts to the color confinement. So, after the Abelian decomposition the color reflection invariance plays the role of the non-Abelian gauge invariance.

As importantly this tells that in QCD the monopole is equivalent to the antimonopole. This is because the monopole quantum number $\pi_{2}\left(S^{2}\right)$ defined by $\hat{n}$ changes the signature under (8), but the magnetic potential $\mathcal{C}_{\mu}$ remains unchanged. So the monopole and anti-monopole are physically undistinguishable in QCD. ${ }^{24,25}$

This should be compared with the Maxwellian electric potential $\mathcal{A}_{\mu}$ (equivalently $A_{\mu}$ ). Unlike $\mathcal{A}_{\mu}$ it changes the signature. So $\mathcal{A}_{\mu}$ and $\mathcal{C}_{\mu}$ (equivalently $A_{\mu}$ and $\tilde{C}_{\mu}$ ) have the negative and positive color charge conjugation quantum number, respectively. Moreover, this tells that $F_{\mu \nu}$ is not color reflection invariant and thus can not be observable, while $H_{\mu \nu}$ is color reflection invariant and is qualified to be an observable.

The above analysis confirms that $\vec{X}_{\mu}$ and $\mathcal{A}_{\mu}$ are not gauge invariant (and thus can not be observables), which is nothing surprising. What is surprising is that the gauge potential contains a color reflection invariant (and thus observable) part $\mathcal{C}_{\mu}$, and that we can separate this part gauge independently by the Abelian projection.

The two potentials $A_{\mu}$ and $\tilde{C}_{\mu}$ have another important difference. Consider the space inversion $\mathrm{P}$ (the parity)

$$
\vec{x} \rightarrow-\vec{x} .
$$

Under this the Maxwellian $A_{\mu}$ (just like the Abelian gauge potential in QED) behaves as an ordinary vector, so that it has negative parity. ${ }^{26}$ But under the space inversion we have

$$
\tilde{C}_{\mu}=-\frac{1}{g}(1-\cos \theta) \partial_{\mu} \phi \rightarrow \tilde{C}_{\mu}^{(p)}
$$




$$
=\frac{1}{g}(1+\cos \theta) \partial_{\mu} \phi
$$

So (9) simply changes the Dirac string in $\tilde{C}_{\mu}$ from the negative $z$-axis to the positive axis, which does not change the monopole physically. This means that $\tilde{C}_{\mu}$ should be interpreted as an axial vector which has positive parity. ${ }^{3}$

From this we conclude that $J^{P C}$ of the electric potential $A_{\mu}$ (or $\mathcal{A}_{\mu}$ ) becomes $1^{--}$while $J^{P C}$ of the magnetic potential $\tilde{C}_{\mu}\left(\right.$ or $\left.\mathcal{C}_{\mu}\right)$ becomes $1^{++}$, where $C$ represents the color (not ordinary) charge conjugation quantum number. This tells that the electric background made of $F_{\mu \nu}$ is not gauge invariant, while the monopole background made of $H_{\mu \nu}$ is so. So we must use the $C P$-invariant topological background in the calculation of the QCD effective action. ${ }^{23,24}$

Moreover, the monopole background should really be understood as the monopole-antimonopole background, because they are gauge equivalent. These are the lessons from the above analysis which we have to keep in mind in the followings.

\section{QCD Effective Action}

To obtain the one-loop effective action we must devide the potential to the classical and quantum parts and integrate out the quantum part in the presence of the classical background. Let us start from ACD and let the background be $\bar{B}_{\mu}$. In this case the effective action is expressed by the gluon and ghost loop deteminants given by $K$ and $M$,

$$
\begin{gathered}
\Delta S=\frac{i}{2} \ln \operatorname{Det} K-i \ln \operatorname{Det} M, \\
\operatorname{Det}^{-1 / 2} K_{\mu \nu}=\operatorname{Det}\left(-g_{\mu \nu} \bar{D}^{2}+2 i g \bar{G}_{\mu \nu}\right), \\
\operatorname{Det} M=\operatorname{Det}\left(-\bar{D}^{2}\right)^{2},
\end{gathered}
$$

where $\bar{D}_{\mu}$ is the covariant derivative defined by the classical background.

Savvidy and others choosed the Savvidy background $\bar{A}_{\mu}{ }^{7-10}$

$$
\bar{G}_{\mu \nu}=\bar{F}_{\mu \nu}, \quad \bar{F}_{\mu \nu}=H \delta_{[\mu}^{1} \delta_{\nu]}^{2},
$$

where $H$ is a constant chromomagnetic field in $z$-direction. In this case the calculation of the functional determinant of the gluon loop integral amounts to finding the energy spectrum of a charged vector field moving around a constant magnetic field, which is given by ${ }^{27}$

$$
E^{2}=2 g H\left(n+\frac{1}{2}-q S_{3}\right)+k^{2},
$$

where $S_{3}$ and $k$ are the spin and momentum of the vector fields in the direction of the magnetic field, and $q= \pm 1$ is the charge (positive and negative) of the vector fields. This is schematically shown in Fig. 2(A). Notice that for both charges the energy spectrum contains negative eigenvalues (tachyonic modes) which violate the causality. 


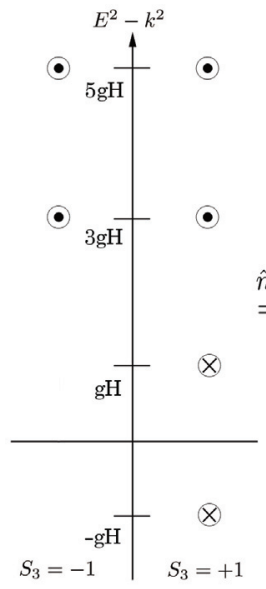

(A)

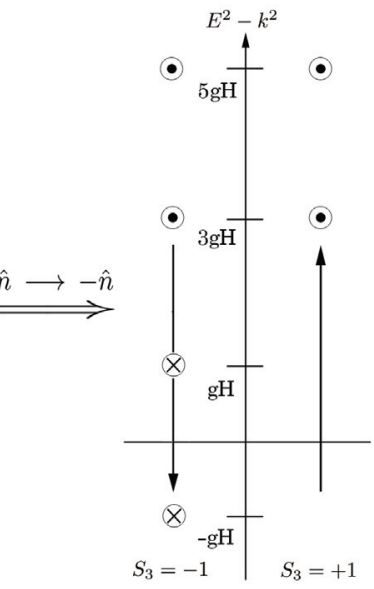

(B)

Fig. 2. The gauge invariant eigenvalues of the gluon functional determinant. Since (A) and (B) should be the C-parity partners, they must have the same eigenvalues for each spin polarization separately. This excludes the lowest two (in particular tachyonic) eigenvalues in both (A) and (B).

From (13) one has the integral expression of the effective action ${ }^{7-10}$

$$
\Delta \mathcal{L}=\lim _{\epsilon \rightarrow 0} \frac{\mu^{2}}{16 \pi^{2}} \int_{0}^{\infty} \frac{d t}{t^{2-\epsilon}} \frac{g H}{\sinh \left(g H t / \mu^{2}\right)}\left[e^{-\frac{2 g H t}{\mu^{2}}}+e^{+\frac{2 g H t}{\mu^{2}}}-2\right],
$$

where $\mu^{2}$ is a dimensional parameter. Clearly the second term has a severe infra-red divergence, but this can be regularized with the standard $\zeta$-function regularization. With this regularization one obtains the SNO effective action ${ }^{7-9}$

$$
\begin{gathered}
\mathcal{L}_{e f f}=-\frac{H^{2}}{2}-\frac{11 g^{2} H^{2}}{48 \pi^{2}}\left(\ln \frac{g H}{\mu^{2}}-c\right)+i \frac{g^{2} H^{2}}{8 \pi}, \\
c=1-\ln 2-\frac{24}{11} \zeta^{\prime}\left(-1, \frac{3}{2}\right)=0.94556 \ldots,
\end{gathered}
$$

which contains the well-known imaginary part which destablizes the SNO vacuum. ${ }^{8}$ Obviously the imaginary part originates from the tachyonic eigenstates.

In general for an arbitrary background the functional determinants are given by $^{7-10}$

$$
\begin{aligned}
\ln \operatorname{Det} K & =2 \ln \operatorname{Det}\left(-\bar{D}^{2}+2 a\right)\left(-\bar{D}^{2}-2 a\right)+2 \ln \operatorname{Det}\left(-\bar{D}^{2}-2 i b\right)\left(-\bar{D}^{2}+2 i b\right), \\
\ln \operatorname{Det} M & =2 \ln \operatorname{Det}\left(-\bar{D}^{2}\right), \\
a & =\frac{g}{2} \sqrt{\sqrt{\bar{G}^{4}+(\bar{G} \bar{G})^{2}}+\bar{G}^{2}}, \quad b=\frac{g}{2} \sqrt{\sqrt{\bar{G}^{4}+(\bar{G} \bar{G})^{2}}-\bar{G}^{2}} .
\end{aligned}
$$


From this we have the well known expression of QCD effective action ${ }^{9,10}$

$$
\begin{aligned}
\Delta S= & i \ln \operatorname{Det}\left(-\bar{D}^{2}+2 a\right)\left(-\bar{D}^{2}-2 a\right)+i \ln \operatorname{Det}\left(-\bar{D}^{2}-2 i b\right)\left(-\bar{D}^{2}+2 i b\right) \\
& -2 i \ln \operatorname{Det}\left(-\bar{D}^{2}\right),
\end{aligned}
$$

and

$$
\begin{aligned}
\Delta \mathcal{L}= & \lim _{\epsilon \rightarrow 0} \frac{1}{16 \pi^{2}} \int_{0}^{\infty} \frac{d t}{t^{3-\epsilon}} \frac{a b t^{2}}{\sinh \left(a t / \mu^{2}\right) \sin \left(b t / \mu^{2}\right)} \\
& \times\left[e^{-2 a t / \mu^{2}}+e^{+2 a t / \mu^{2}}+e^{+2 i b t / \mu^{2}}+e^{-2 i b t / \mu^{2}}-2\right] .
\end{aligned}
$$

Notice that the first four terms are the gluon loop contribution, but the last term comes from the ghost loop. When $a=g H$ and $b=0$, this becomes identical to (14). Notice that the second and fourth terms have a severe infra-red divergence.

There are two critical defects in the old calculations. First, the Savvidy background (12) is neither gauge invariant nor parity conserving, as we have emphasized. Second, the gauge invariance is completely overlooked in the calculation of the functional determinants. In particular, the color reflection invariance (the C-parity) is not correctly implemented in the old calculations.

To show how to calculate the effective action correctly, we choose the monopole background $\bar{C}_{\mu}$ obtained from the gauge independent Abelian decomposition (3),

$$
\bar{G}_{\mu \nu}=\bar{H}_{\mu \nu}, \quad \bar{H}_{\mu \nu}=H \delta_{[m}^{1} \delta_{n]}^{2} .
$$

This should be compared with the Savvidy background (12) which is not gauge invariant nor parity conserving. To be general, however, we will let $\bar{H}_{\mu \nu}$ arbitrary but constant in the following.

Now, we have to integrate out the colored gluons gauge invariantly, imposing the color reflection invariance invariance. To do that, consider the case $a=g H$ and $b=0$ shown in Fig. 2 again. Clearly the color reflection (the C-parity) changes (A) to (B), so that they are gauge equivalent. But since this $C$-parity does not change the spin of the valence gluons, we must require the physical eigenvalues to be invariant under the C-parity for each spin polarization separately.

Now, obviously the lowest two eigenvalues for both $S_{3}=+1$ in (A) and $S_{3}=-1$ in (B) do not satisfy this requirement, so that they must be discarded. This, of course, removes the tachyonic states. This is the C-projection which restores the gauge invariance in the gluon loop integral. This neglect of gauge invariance is the critical mistake of the conventional calculations. ${ }^{7-10}$

Notice that the C-parity here plays exactly the same role as the G-parity in string theory. It is well known that the GSO projection (the G-projection) restores the supersymmetry and modular invariance in NSR string by projecting out the tachyonic vacuum. ${ }^{11,12}$ Just like the G-projection in string, the C-projection in $Q C D$ removes the tachyonic modes and restores the gauge invariance of the effective action. 
Exactly the same argument applies to $\operatorname{Det}\left(-\bar{D}^{2} \pm 2 i b\right)$ in (17). Here again they are the C-parity counterpart of each other, so that theymust have exactly the same contribution. This tells that the correct effective action is given by ${ }^{24}$

$$
\Delta S=2 i \ln \operatorname{Det}\left(-\bar{D}^{2}+2 a\right)+2 i \ln \operatorname{Det}\left(-\bar{D}^{2}-2 i b\right)-2 i \ln \operatorname{Det}\left(-\bar{D}^{2}\right),
$$

and

$$
\Delta \mathcal{L}=\lim _{\epsilon \rightarrow 0} \frac{1}{8 \pi^{2}} \int_{0}^{\infty} \frac{d t}{t^{3-\epsilon}} \frac{a b t^{2} / \mu^{4}}{\sinh \left(a t / \mu^{2}\right) \sin \left(b t / \mu^{2}\right)}\left[e^{-\frac{2 a t}{\mu^{2}}}+e^{+\frac{2 i b t}{\mu^{2}}}-1\right] .
$$

This is the new integral expression of QCD effective action, which should be compared with (18). Obviously the C-projection makes (21) gauge invariant. As importantly, it removes the infra-red divergence of (18). this tells that, we do not need the $\zeta$-function regularization (or any regularization) if we calculate the effective action correctly.

At the first thought this might be surprising, but actually is not so. The gauge invariance implies the confinement. This implies the generation of a mass parameter, which should make the theory infra-red finite. So it is natural that the gauge invariance makes (21) infra-red finite.

When $a=0$ or $b=0$, the integration is straightforward. For pure magnetic background we have

$$
\Delta \mathcal{L}=\lim _{\epsilon \rightarrow 0} \frac{a / \mu^{2}}{8 \pi^{2}} \int_{0}^{\infty} \frac{d t}{t^{2-\epsilon}} \frac{\exp \left(-2 a t / \mu^{2}\right)}{\sinh \left(a t / \mu^{2}\right)}
$$

So that ${ }^{28,29}$

$$
\mathcal{L}_{\text {eff }}=-\frac{a^{2}}{2 g^{2}}-\frac{11 a^{2}}{48 \pi^{2}}\left(\ln \frac{a}{\mu^{2}}-c^{\prime}\right),
$$

where $c^{\prime}$ is a constant. This is identical to the SNO effective action (16), except that here there is no imaginary part. This, of course, assures the stability of the monopole condensation.

For the pure electric background we have

$$
\Delta \mathcal{L}=\lim _{\epsilon \rightarrow 0} \frac{b / \mu^{2}}{8 \pi^{2}} \int_{0}^{\infty} \frac{d t}{t^{2-\epsilon}} \frac{\exp \left(+2 i b t / \mu^{2}\right)}{\sin \left(b t / \mu^{2}\right)}
$$

so that ${ }^{28,29}$

$$
\mathcal{L}_{e f f}=\frac{b^{2}}{2 g^{2}}+\frac{11 b^{2}}{48 \pi^{2}}\left(\ln \frac{b}{\mu^{2}}-c^{\prime}\right)-i \frac{11 b^{2}}{96 \pi} .
$$

To summarize we have

$$
\mathcal{L}_{e f f}= \begin{cases}-\frac{a^{2}}{2 g^{2}}-\frac{11 a^{2}}{48 \pi^{2}}\left(\ln \frac{a}{\mu^{2}}-c^{\prime}\right), & \quad b=0 \\ \frac{b^{2}}{2 g^{2}}+\frac{11 b^{2}}{48 \pi^{2}}\left(\ln \frac{b}{\mu^{2}}-c^{\prime}\right) & \\ -i \frac{11 b^{2}}{96 \pi}, & a=0\end{cases}
$$


Notice that when $a=0$ the imaginary part has a negative signature, which implies the pair annihilation of gluons. ${ }^{29,30}$

This must be contrasted with the QED effective action where the electron loop integral generates a positive imaginary part. ${ }^{31,32}$ The positive imaginary part in QED means the pair creation which generates the screening. On the other hand in QCD we must have the anti-screening to explain the asymptotic freedom, and the negative imaginary part is what we need for the asymptotic freedom. ${ }^{28-30}$

Observe that (23) and (25) are related by the electric-magnetic duality. ${ }^{28}$ We can obtain one from the other simply by replacing $a$ with $-i b$ and $b$ with $i a$. This duality, which states that the effective action should be invariant under the replacement

$$
a \rightarrow-i b, \quad b \rightarrow i a,
$$

was first discovered in the QED effective action. ${ }^{32}$ But subsequently this duality has also been shown to exist in the QCD effective action. ${ }^{28,29}$ This tells that the duality should be regarded as a fundamental symmetry of the effective action of gauge theory, Abelian and non-Abelian. The importance of this duality is that it provides a very useful tool to check the self-consistency of the effective action. The fact that (23) and (25) are related by the duality assures that they are self-consistent.

\section{Monopole Condensation}

The effective action (23) generates the much desired dimensional transmutation in QCD. From (23) we have the following effective potential

$$
V=\frac{H^{2}}{4}\left[1+\frac{11 g^{2}}{24 \pi^{2}}\left(\ln \frac{g H}{\mu^{2}}-c\right)\right] .
$$

From this we define the running coupling $\bar{g}$ by ${ }^{28,29}$

$$
\left.\frac{\partial^{2} V}{\partial H^{2}}\right|_{H=\bar{\mu}^{2}}=\frac{1}{2} \frac{g^{2}}{\bar{g}^{2}}
$$

and obtain the following $\beta$-function

$$
\beta(\bar{\mu})=\bar{\mu} \frac{\partial \bar{g}}{\partial \bar{\mu}}=-\frac{11 \bar{g}^{3}}{24 \pi^{2}} .
$$

This is exactly the same $\beta$-function that one obtained from the perturbative QCD. ${ }^{33}$ In terms of the running coupling the renormalized potential is given by

$$
V_{\text {ren }}=\frac{H^{2}}{4}\left[1+\frac{11 \bar{g}^{2}}{24 \pi^{2}}\left(\ln \frac{H}{\bar{\mu}^{2}}-\frac{3}{2}\right)\right],
$$

which generates a non-trivial local minimum at

$$
\langle H\rangle=\frac{\bar{\mu}^{2}}{\bar{g}} \exp \left(-\frac{24 \pi^{2}}{11 \bar{g}^{2}}+1\right) .
$$

This is nothing but the desired magnetic condensation. The corresponding effective potential is plotted in Fig. 3, where we have assumed $\bar{\alpha}_{s}=1$ and $\bar{\mu}=1$. 


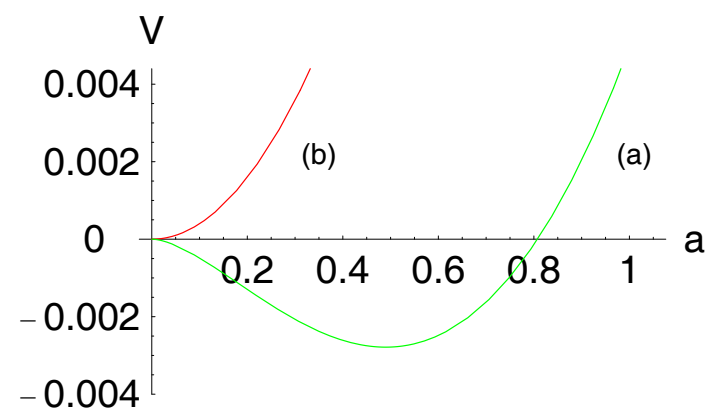

Fig. 3. The effective potential of SU(2) QCD in the pure magnetic background. Here (a) is the effective potential and (b) is the classical potential.

Nielsen and Olesen have suggested that the existence of the tachyonic modes is closely related to the asymptotic freedom. ${ }^{8}$ Our analysis tells that this is not true. Obviously (30) is consistent with the stable magnetic condensation.

In retrospect it is not surprising that the gauge invariance plays the crucial role in the stability of the monopole condensation. From the beginning the gauge invariance has been the main motivation for the confinement in QCD. It is this gauge invariance which forbids colored objects from the physical spectrum of QCD. This necessitates the confinement of color. So it is only natural that the gauge invariance assures the stability of the magnetic condensation, and thus the confinement of color. Of course, in ECD and ACD the gauge invariance translates to the color reflection invariance, which can be implemented by the C-projection.

To prove that the monopole condensation is the true minimum of the effective potential, of course, we have to integrate the effective action (21) for arbitrary $a$ and $b$. This is not easy. Even in the "simpler" QED, the integration of the effective action has been completed only recently. ${ }^{32}$ But we can do the integration for arbitrary $a b \neq 0$, and prove that the monopole condensation is the true minimum of the effective potential. ${ }^{34}$

An unavoidable consequence of the monopole condensation is the existence of two magnetic glueballs, the $0^{++}$and $1^{++}$modes of the vacuum fluctuation, which are not made of the valence gluons. This is because the monopole condensation induces two scales, the correlation length of the monopoles and the penetration length of the color flux. ${ }^{3}$ Obviously they are similar to the Higgs scalar and the massive photon that we have in spontaneous symmetry breaking, and it is very difficult to avoid these modes if we believe in the monopole condensation. The challenge now is to confirm (or disprove) the existence of these magnetic glueballs experimentally.

\section{Discussion}

Establishing the monopole condensation in QCD has been extremely difficult to attain. Savvidy and many others had the correct idea, but made serious mistakes 
in implementing the idea. The old calculations have two critical defects. First, they have chosen the wrong background which is neither gauge invariant nor parity conserving. This is the problem of the SNO background. Second, they have failed to impose the gauge invariance in the gluon loop integral. This is the problem of the infra-red instability of the effective action.

The first problem arises because the old calculations neglected the fact that there are actually two possible magnetic backgrounds in QCD, the SNO background and the monopole background, but only the monopole background qualifies to be the QCD vacuum because only this background is gauge invariant and parity conserving. This is because the old calculations ignored the fact that the restricted potential has a dual structure.

The second problem, the infra-red instability of the SNO vacuum, originates from the fact that the old calculations failed to implement the gauge invariance in the calculation of the gluon functional determinant properly. In this paper we have shown how the C-projection makes the functional determinant gauge invariant and removes the tachyonic modes which cause the effective action unstable. This assures the stable monopole condensation.

The Abelian decomposition (3) provides a perfect setting to calculate the oneloop effective action of QCD correctly. This decomposition separates not only colored potential but also the monopole potential gauge independently, and thus allows us to choose the gauge invariant monopole background. Moreover it tells exactly how each term transforms under the gauge transformation (the color reflection), and tells how to implement the gauge invariance in the calculation of the effective action with the color reflection.

It is not surprising that the gauge invariance plays the key role to generate the stable monopole condensation. From the beginning the gauge invariance has been the main motivation for the confinement. It is this gauge invariance which forbids any colored object from the physical spectrum of QCD. So it is only natural that the gauge invariance assures the stability of the monopole condensation, and thus the confinement of color.

The existence of the tachyonic modes which caused the infra-red instability of the effective action has been a headache, the Gordian knot, in QCD. In fact it has been thought that this infra-red instability is an essential characteristic, a sacred feature, of QCD which comes from the absence of a mass parameter. But in this paper we have shown that the tachyons are an unphysical mirage which should not have been there in the first place. They disappear when we calculate the effective action correctly.

As we have emphasized, in physics tachyons appear when we do something improper or choose something unphysical. In spontaneous symmetry breaking we have the tachyons in the false vacuum. But they disappear if we choose the correct vacuum. Similarly, in NSR string theory we have the tachyonic vacuum when we do not impose the modular invariance and supersymmetry with the G-projection. ${ }^{11,12}$ Here we have the same situation. The tachyons appear when we do not implement 
the gauge invariance to the gluon functional determinant correctly. This tells that there is nothing mysterious about the tachyons in QCD.

Notice, however, that it is not just tachyons which violate the gauge invariance in QCD. Clearly Fig. 2 shows that the lowest two eigenvalues which include the nontacyonic modes violate the gauge invariance, and should be projected out by the C-projection. This is very important because, had we excluded only the tachyons, we would not have had the gauge invariance and thus the correct effective action.

We can compare the monopole condensation with the Abelian dominance, which has been a very popular in lattice QCD. ${ }^{4,5}$ Our result tells that the Abelian dominance is indeed correct, because the monopole is in the Abelian part. On the other hand, the "Abelian dominance" based on the maximal Abelian gauge is clearly misleading and confusing. First, it does not tell what is the Abelian part. Second, although the gauge independent Abelian projection (1) does tell what is the Abelian part, it is made of two terms. So we have to tell which causes the confinement. The Abelian dominance does not tell what is this.

It must be emphasized, however, that the monopole condensation should really be understood as the monopole-antimonopole condensation. This is because in QCD the monopole and anti-monopole are gauge equivalent, because they are the C-parity partners. $^{24,25}$

At this point one may ask what is wrong with the SNO effective action. Clearly it is not the effective action of QCD but the effective action of a U(1) gauge theory coupled to a massless charged vector field which has no color reflection invariance. This is not QCD, but actually a sick theory. It is well known that such theory is ill-defined. So there is nothing wrong with the SNO effective action, except that it is the effective action of a sick theory. And the problems of the SNO vacuum are the symptoms of this sickness.

It is truly remarkable (and surprising) that the principles of quantum field theory allow us to demonstrate confinement within the framework of QCD. There has been a proof of confinement in a supersymmetric QCD. ${ }^{35}$ Our analysis shows that we can actually establish the existence of the confinement phase within the conventional QCD, with the existing principles of quantum field theory. This should be interpreted as a most spectacular triumph of quantum field theory itself.

In this paper we have neglected the quarks. We simply remark that the quarks, just as in the asymptotic freedom, tend to destabilize the monopole condensation. But if the number of quarks are small enough, the condensation remains stable. In fact we can show that the stability puts exactly the same constraint on the number of quarks as the asymptotic freedom. ${ }^{36}$

In real QCD, of course, we have to deal with $\mathrm{SU}(3)$. We can do this, and the generic feature of the $\mathrm{SU}(2) \mathrm{QCD}$, in particular the dimensional transmutation by the monopole condensation, remains the same. ${ }^{36}$ 


\section{Acknowledgments}

This work is supported in part by the Basic Science Research Program through the National Research Foundation (Grant 2012-002-134) of the Ministry of Science and Technology and by Konkuk University.

\section{References}

1. Y. Nambu, Phys. Rev. D10, 4262 (1974); S. Mandelstam, Phys. Rep. 23C, 245 (1976); A. Polyakov, Nucl. Phys. B120, 429 (1977).

2. Y. M. Cho, Phys. Rev. D21, 1080 (1980). See also Y. S. Duan and M. L. Ge, Sci. Sinica 11,1072 (1979).

3. Y. M. Cho, Phys. Rev. Lett. 46, 302 (1981); Phys. Rev. D23, 2415 (1981).

4. G. 't Hooft, Nucl. Phys. B190, 455 (1981).

5. A. Kronfeld, G. Schierholz and U. Wiese, Nucl. Phys. B293, 461 (1987); A Kronfeld, M. Laursen, G. Schierholz and U. Wiese, Phys. Lett. B198, 516 (1987); T. Suzuki and I. Yotsuyanagi, Phys. Rev. D42, 4257 (1990).

6. S. Coleman and E. Weinberg, Phys. Rev. D7, 1888 (1973).

7. G. K. Savvidy, Phys. Lett. B71, 133 (1977).

8. N. Nielsen and P. Olesen, Nucl. Phys. B144, 485 (1978); B160, 380 (1979); C. Rajiadakos, Phys. Lett. B100, 471 (1981).

9. W. Dittrich and M. Reuter, Phys. Lett. B128, 321, (1983); B144, 99 (1984); M. Reuter, M. G. Schmidt and C. Schubert, Ann. Phys. 259, 313 (1997).

10. A. Yildiz and P. Cox, Phys. Rev. D21, 1095 (1980); M. Claudson, A. Yilditz and P. Cox, Phys. Rev. D22, 2022 (1980); J. Ambjorn and R. Hughes, Phys. Lett. B113, 305 (1982).

11. F. Gliozzi, J. Scherk and D. Olive, Nucl. Phys. B122, 253 (1977).

12. See, e. g., M. Green, J. Schwarz and E. Witten, Superstring Theory, Vol. I (Cambridge University Press) 1987; M. Kaku, Introduction to Superstrings (Springer-Verlag) 1988.

13. Y. M. Cho, Phys. Rev. D62, 074009 (2000).

14. S. Kato, K. Kondo, T. Murakami, A. Shibata, T. Shinohara and S. Ito, Phys. Lett. B632, 326 (2006); B645, 67 (2007); B653, 101 (2007); B669, 107 (2008).

15. N. Cundy, Y. M. Cho and W. Lee, PoS LATTICE2012, 212 (2012).

16. T. T. Wu and C. N. Yang, Phys. Rev. D12, 3845 (1975).

17. Y. M. Cho, Phys. Rev. Lett. 44, 1115 (1980).

18. L. Faddeev and A. Niemi, Phys. Rev. Lett. 82, 1624 (1999); Phys. Lett. B449, 214 (1999).

19. S. Shabanov, Phys. Lett. B458, 322 (1999); B463, 263 (1999); H. Gies, Phys. Rev. D63, 125023 (2001).

20. R. Zucchini, Int. J. Geom. Meth. Mod. Phys. 1, 813 (2004).

21. B. de Witt, Phys. Rev. 162, 1195 (1967); 1239 (1967).

22. See for example, C. Itzikson and J. Zuber, Quantum Field Theory (McGraw-Hill) 1985; M. Peskin and D. Schroeder, An Introduction to Quantum Field Theory (AddisonWesley) 1996.

23. W. S. Bae, Y. M. Cho and S. W. Kim, Phys. Rev. D65, 025005 (2001).

24. Y. M. Cho, Franklin H. Cho and J. H. Yoon, Phys. Rev. D87, 085025 (2013).

25. Y. M. Cho, Phys. Lett. B115, 125 (1982).

26. See for example, J. J. Sakurai, Invariance Principle and Elementary Particles (Princeton University Press) 1964, p. 113.

27. W. Tsai and A. Yildiz, Phys. Rev. D4, 3643 (1971); T. Goldman and W. Tsai, ibid, 3648 (1971). 
28. Y. M. Cho and D. G. Pak, Phys. Rev. D65, 074027 (2002); Y. M. Cho, H. W. Lee and D. G. Pak, Phys. Lett. B525, 347 (2002).

29. Y. M. Cho, M. L. Walker and D. G. Pak, JHEP 05, 073 (2004); Y. M. Cho and M. L. Walker, Mod. Phys. Lett A19, 2707 (2004).

30. V. Schanbacher, Phys. Rev. D26, 489 (1982).

31. J. Schwinger, Phys. Rev. 82, 664 (1951).

32. Y. M. Cho and D. G. Pak, Phys. Rev. Lett. 86, 1947 (2001); 91, 039151; W. S. Bae, Y. M. Cho and D. G. Pak, Phys. Rev. D64, 017303 (2001).

33. D. Gross and F. Wilczek, Phys. Rev. Lett. 30, 1343 (1973); H. Politzer, Phys. Rev. Lett. 30, 1346 (1973).

34. Y. M. Cho and D. G. Pak, hep-th/0006051. See also Y. M. Cho and D. G. Pak, in Proceedings of TMU-Yale Symposium on Dynamics of Gauge Fields, edited by T. Appelquist and H. Minakata (Universal Academy Press, Tokyo) 1999.

35. N. Seiberg and E. Witten, Nucl. Phys. B426, 19 (1994); B431, 484 (1994).

36. Y. M. Cho, to be published. 